\title{
Inhibition potentiates the synchronizing action of electrical synapses
}

\author{
Benjamin Pfeuty ${ }^{1 a}$, David Golomb ${ }^{2}$, Germán Mato ${ }^{3}$ and David Hansel ${ }^{1,4, *}$ \\ 1. Laboratoire de Neurophysique et Physiologie and CNRS, UMR 8119, Université Paris Descartes, France \\ 2. Department of Physiology and Zlotowski Center for Neuroscience, Faculty of Health Sciences, Ben Gurion University of the Negev, \\ Israel \\ 3. Comisión Nacional de Energia Atómica and CONICET, Centro Atómico Bariloche and Instituto Balseiro, Universidad Nacional de \\ Cuyo, Argentina \\ 4. Interdisciplinary Center for Neural Computation, The Hebrew University, Israel
}

Edited by: Misha Tsodyks, Weizmann Institute of Science, Israel

Reviewed by: Udo A. Ernst, University of Bremen, Germany

Mate Lengyel, Collegium Budapest Institute for Advanced Study, Hungary

\begin{abstract}
In vivo and in vitro experimental studies have found that blocking electrical interactions connecting GABAergic interneurons reduces oscillatory activity in the $\gamma$ range in cortex. However, recent theoretical works have shown that the ability of electrical synapses to promote or impede synchrony, when alone, depends on their location on the dendritic tree of the neurons, the intrinsic properties of the neurons and the connectivity of the network. The goal of the present paper is to show that this versatility in the synchronizing ability of electrical synapses is greatly reduced when the neurons also interact via inhibition. To this end, we study a model network comprising two-compartment conductance-based neurons interacting with both types of synapses. We investigate the effect of electrical synapses on the dynamical state of the network as a function of the strength of the inhibition. We find that for weak inhibition, electrical synapses reinforce inhibition-generated synchrony only if they promote synchrony when they are alone. In contrast, when inhibition is sufficiently strong, electrical synapses improve synchrony even if when acting alone they would stabilize asynchronous firing. We clarify the mechanism underlying this cooperative interplay between electrical and inhibitory synapses. We show that it is relevant in two physiologically observed regimes: spike-to-spike synchrony, where neurons fire at almost every cycle of the population oscillations, and stochastic synchrony, where neurons fire irregularly and at a rate which is substantially lower than the frequency of the global population rhythm.
\end{abstract}

Keywords: gap-junction, inhibitory interneurons, cortical dynamics, network model, synchrony, gamma oscillations

\section{INTRODUCTION}

Electrical synapses are frequently found to connect GABAergic interneurons in the central nervous system. This is for instance the case in the neocortex (Fukuda and Kosaka, 2000; Galarreta and Hestrin 1999; Gibson et al., 1999), in the striatum (Kita et al., 1990), the hippocampus (Venance et al., 2000), the cerebellum (Mann-Metzer and Yarom, 1999), and the reticular thalamic nucleus (Landisman et al., 2002). In the neocortex, low threshold spiking (LTS) GABAergic interneurons interact mostly via electrical synapses whereas both electrical and GABAergic synapses are found between fast spiking (FS) interneurons (Beierlein et al., 2003). Multipolar bursting interneurons in layer $2 / 3$ in frontal and somatosensory cortex are also connected by both kinds of synapses (Blatow et al., 2003).

* Correspondence: David Hansel, Laboratoire de Neurophysique et Physiologie and CNRS, UMR 8119, Université Paris Descartes, 45 rue des Saints Pères, 75270 Paris Cedex 06, France. e-mail: david.hansel@biomedicale.univ-paris5.fr

a Benjamin Pfeuty's present address is Department of Pure and Applied Sciences, University of Tokyo, Tokyo 153-8902, Japan.

Received: 21 Sep. 2007; paper pending published: 11 0ct. 2007; accepted: 15 0ct. 2007; published online: 2 Nov. 2007.

Full citation: Frontiers in Computational Neuroscience (2007) 1:8

doi: 10.3389/neuro.10/008.2007

Copyright: () 2007 Pfeuty, Golomb, Mato and Hansel. This is an open-access article subject to an exclusive license agreement between the authors and the Frontiers Research Foundation, which permits unrestricted use, distribution, and reproduction in any medium, provided the original authors and source are credited.
It has been proposed that electrical synapses are involved in the generation of synchronous rhythm in the hippocampus and in the neocortex (Bartos et al., 2007; Fricker and Miles, 2001; LeBeau et al., 2003; Whittington and Traub, 2003). In hippocampal and neocortical slices, $\gamma$ oscillations are abolished by gap junction blockers (LeBeau et al., 2002; Traub et al., 2001). These oscillations are also reduced in transgenic Cx-36- knockout mice both in vitro and in vivo (Buhl et al., 2003; Deans et al., 2001; Hormuzdi et al., 2001). Interestingly, $\gamma$-oscillations are also abolished by the $\mathrm{GABA}_{\mathrm{A}}$ antagonist bicuculline (LeBeau et al., 2002; Whittington et al., 1995).

Recent theoretical studies have shown that the ability of electrical synapses to promote synchrony depends on the firing frequency of the neurons (Chow and Kopell, 2000; Lewis and Rinzel, 2003; Pfeuty et al., 2003), on the intrinsic properties of the neurons (Mancilla et al., 2007; Pfeuty et al., 2003; Saraga et al., 2006), and on the location of these synapses on the dendritic trees (Pfeuty et al., 2005; Saraga et al., 2006). In particular, one question is whether synapses on the dendritic tree of interneurons at distances as far as 100-200 $\mu \mathrm{m}$ from the soma (Fukuda and Kosaka, 2000; Fukuda et al., 2006; Kosaka and Hama, 1985; Sloper, 1972; Szabadics et al., 2001) contribute to the emergence of rhythmic activity in networks of inhibitory interneurons.

The goal of the present paper is to show that when combined with sufficiently strong inhibitory synapses, electrical synapses always promote synchrony even in the case they would impede it in the absence 
of inhibition. To this end, we study the dynamics of a large network of two-compartment conductance-based neurons interacting with both electrical and inhibitory interactions. We investigate how electrical synapses affect the dynamical state of this network as a function of the conductance of the inhibitory synapses. For weak inhibition, electrical synapses improve synchrony only when they promote synchrony when they are alone. In contrast, when inhibition is sufficiently strong, we find that they always reinforce the rhythm and its robustness to noise. We show that this cooperative interplay between electrical synapses and inhibition is relevant in two distinct physiological observed regimes of synchrony: (1) during spike-to-spike synchrony where neurons fire at almost every cycle of the population oscillations and (2) during stochastic synchrony where the firing rate of the neurons is significantly lower that the frequency of the global oscillation (Brunel and Hakim, 1999; Brunel and Hansel, 2006; Tiesinga and Jose, 2000). In the latter case, the presence of electrical synapses significantly improves the robustness of synchrony at low firing rates and high synaptic conductances where inhibition alone often fails to synchronize. Finally, we discuss the mechanism of this cooperative interplay and its relevance in the emergence of $\gamma$ rhythm.

\section{MATERIAL AND METHODS}

\section{Single neuron dynamics}

Our conductance-based neuronal model is a two-compartments (soma, dendrite) generalization of the neuronal model devised by Wang and Buzsáki (1996). The somatic compartment incorporates a leak current $I_{\mathrm{L}}$, a transient sodium current $I_{\mathrm{Na}}$ and a delayed rectifier potassium current $I_{\mathrm{Kdr}}$. The dendritic compartment is passive. It receives an external input $I_{\text {ext }}$. The membrane potential of the soma, $V^{\mathrm{s}}$, and the dendrite, $V^{\mathrm{d}}$, obey the current balance equations:

$C \frac{\mathrm{d} V^{\mathrm{s}}}{\mathrm{d} t}=-I_{\mathrm{L}}-I_{\mathrm{Na}}-I_{\mathrm{Kdr}}-g_{\mathrm{c}}\left(V^{\mathrm{s}}-V^{\mathrm{d}}\right)+I_{\text {noise }}$

$C \frac{\mathrm{d} V^{\mathrm{d}}}{\mathrm{d} t}=-I_{\mathrm{L}, \mathrm{d}}-g_{\mathrm{c}}\left(V^{\mathrm{d}}-V^{\mathrm{s}}\right)+I_{\mathrm{ext}}$

The leak and the voltage gated currents are given by: $I_{\mathrm{L}}=$ $-g_{\mathrm{L}}\left(V^{\mathrm{s}}-V_{\mathrm{L}}\right), I_{\mathrm{L}, \mathrm{d}}=-g_{\mathrm{L}}\left(V^{\mathrm{d}}-V_{\mathrm{L}}\right), I_{\mathrm{Na}}=g_{\mathrm{Na}} m_{\infty}^{3} h\left(V^{\mathrm{s}}-V_{\mathrm{Na}}\right)$ and $I_{K}=g_{K} n^{4}\left(V^{\mathrm{s}}-V_{\mathrm{K}}\right)$ where the gating variables $h$ and $n$ satisfy:

$\frac{\mathrm{d} x}{\mathrm{~d} t}=\alpha_{x}\left(V^{\mathrm{s}}\right)(1-x)-\beta_{x}\left(V^{\mathrm{s}}\right) x$

with $x=h, n, \alpha_{h}(V)=0.21 e^{-(V+58) / 20}, \beta_{h}(V)=3 /\left(1+e^{-(V+28) / 10}\right)$ $\alpha_{n}(V)=0.03(V+34) /\left(1-e^{-(V+34) / 10}\right)$, and $\beta_{n}(V)=0.375 e^{-(V+44) / 80}$ The activation function $m_{\infty}$, is given by: $m_{\infty}(V)=\alpha_{m}(V) /\left(\alpha_{m}(V)+\right.$ $\left.\beta_{m}(V)\right)$, with $\alpha_{m}(V)=0.1(V+35) /\left(1-e^{-(V+35) / 10}\right)$ and $\beta_{m}(V)=$ $4 e^{-(V+60) / 18}$. The other parameters of the model are $g_{\mathrm{Na}}=35 \mathrm{mS} / \mathrm{cm}^{2}$, $V_{\mathrm{Na}}=55 \mathrm{mV}, g_{\mathrm{K}}=9 \mathrm{mS} / \mathrm{cm}^{2}, V_{\mathrm{K}}=-75 \mathrm{mV}, g_{\mathrm{L}}=0.1 \mathrm{mS} / \mathrm{cm}^{2}$, $g_{c}=0.3 \mathrm{mS} / \mathrm{cm}^{2}, V_{\mathrm{L}}=-65 \mathrm{mV}$, and $C=1 \mu \mathrm{F} / \mathrm{cm}^{2}$.

When the external current, $I_{\mathrm{ext}}$, is sufficiently large the neuron discharges action potentials periodically. The relationship between $I_{\mathrm{ext}}$ and the frequency, $f$, of the discharge is plotted in Figure 1A. The current $I_{\text {noise }}$ represents the effect of the intrinsic and the synaptic noise in the neuron. It is modeled as a Gaussian white noise current with a zero mean and a standard deviation, $\sigma$.

\section{The network}

The network consists of $N$ neurons interacting via electrical and inhibitory synapses. The connectivity is random. Unless specified otherwise, each neuron is connected on average to $K_{\text {gap }}=10$ and $K_{\text {inh }}=50$ other neurons via electrical synapses and via inhibitory synapses, respectively, in line with experimental data (Amitai et al., 2002; Sik et al., 1995).

We assume that the inhibitory synapses are located on the dendritic compartment. Therefore, we incorporate their effect by adding a synaptic
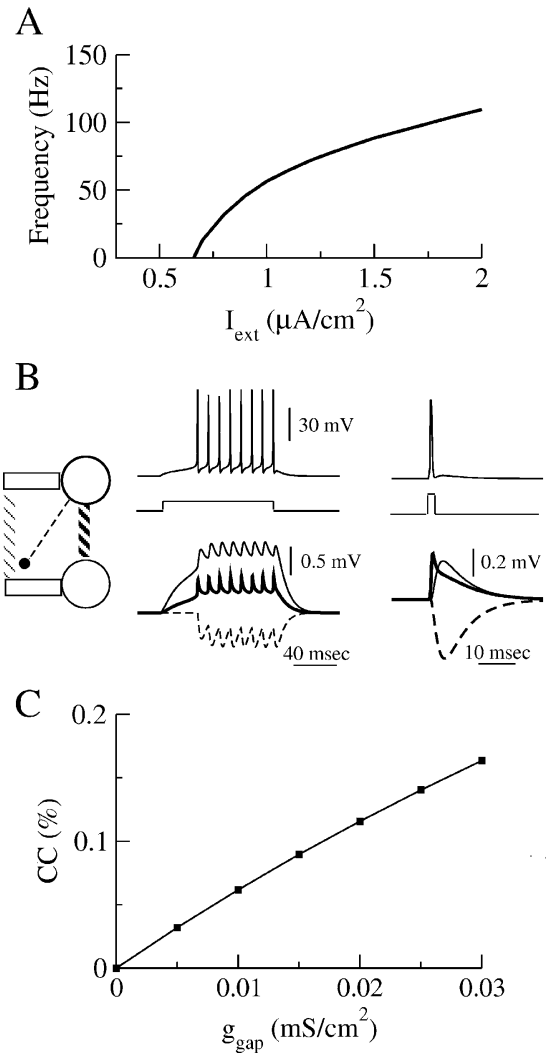

Figure 1. Properties of the model. (A) The relationship between the discharge frequency and the injected current, $I_{\text {ext }}$, (F-I curve) for our model neuron. (B) Post-synaptic potentials evoked by a train of action potentials (left top panel) or by one presynaptic action potential (right top panel) following a long or short transient pulse of current. Post-synaptic potentials are shown (bottom panels) for the three types of interaction in a two-neurons system represented on the left diagram: the neurons interact via an electrical synapse connecting their soma $\left(g_{\text {gap }}=0.005 \mathrm{mS} / \mathrm{cm}^{2}\right.$, solid thick lines); the neurons interact via an electrical synapse connecting their dendritic compartments $\left(g_{\text {gap }}=0.02 \mathrm{mS} / \mathrm{cm}^{2}\right.$, solid thin lines); The neurons are coupled by inhibitory synapses located on their dendrite $\left(g_{\text {inh }}=0.05 \mathrm{mS} / \mathrm{cm}^{2}\right.$, dashed lines). (C) Coupling coefficient (CC) measured at the soma between two neurons coupled with electrical synapses on the dendritic compartments.

current to the right-hand side of Equation (2):

$I_{\text {inh }}(t)=-g_{\text {inh }} s(t)\left(V^{\mathrm{d}}(t)-V_{\text {inh }}\right)$

where $g_{\text {inh }}$ is a constant conductance, $V_{\text {inh }}=-75 \mathrm{mV}$ is the reversal potential of the inhibitory synapses, and the function $s(t)$ satisfies the dynamics:

$\frac{\mathrm{d} s}{\mathrm{~d} t}=50\left(1+\tan h\left(V^{\mathrm{s}} / 4\right)\right)(1-s)-s / \tau_{\text {inh }}$

with $\tau_{\text {inh }}=3 \mathrm{~ms}$.

In this work, we study the effect on synchrony of the electrical synapses located between the somatic or between the dendritic compartments of two neurons. In the first case, the effect of neuron $j$ on neuron $i$ is modeled by a synaptic current

$I_{\text {gap }}=g_{\text {gap }}\left(V_{i}^{\mathrm{s}}-V_{j}^{\mathrm{s}}\right)$

added to the right-hand side of Equation (1) for neuron $j$. Similarly, if the electrical synapse couples the dendrites of the neurons, a current

$I_{\text {gap }}=g_{\text {gap }}\left(V_{i}^{\mathrm{d}}-V_{j}^{\mathrm{d}}\right)$

2 
is added to the right-hand side of Equation (2) for neuron $j$. The inhibitory postsynaptic potentials and the spikelets associated with each type of interaction are illustrated in Figure 1B. Note that the time course of the spikelets mediated by electrical synapses coupling the dendritic compartment are broadened by the dendritic filtering.

The strength of an electrical synapse can be quantified through the coupling coefficient (CC) defined as the ratio between the voltage changes in the postsynaptic neuron and in the presynaptic neuron as the latter is injected with a constant current. Experimental studies in cortex report typical values of CCs on the order of or smaller than 0.15 (Amitai et al., 2002; Gibson et al., 1999; Venance et al., 2000). The CCs used in our model have similar values, as shown in Figure 1C.

Note that $g_{\text {gap }}$ (resp. $g_{\text {inh }}$ ) characterizes the effect of one single electrical (resp. inhibitory) synapse. The values of these conductances have to be multiplied by the average connectivities, $K_{\text {gap }}$ or $K_{\text {inh }}$, to estimate the strength of the synaptic currents the neurons receive in the network.

\section{Numerical simulations of the model network}

We simulated our model network using the second order Runge-Kutta integration scheme with fixed time step: $\delta t=0.01 \mathrm{~ms}$. Average quantities such as the firing rate, the CV of the interspike interval, and the measure of synchrony, $\chi$ (see below) were computed over a time period of 1 second after discarding a transient of $500 \mathrm{~ms}$.

Measure of synchrony. We quantify the degree of synchrony in the network following (Golomb and Rinzel, 1994; Hansel and Sompolinsky, 1992, 1996). We denote by $\bar{V}(t)$ the population average membrane potential:

$\bar{V}(t)=\frac{1}{N} \sum_{i=1}^{N} V_{i}(t)$

and by $\sigma_{\bar{V}}$ and $\sigma_{V_{i}}$ the standard deviation of the temporal fluctuations of $\bar{V}$ and of the individual voltage of neuron $i$, respectively:

$\sigma_{\bar{V}}^{2}=\left\langle[\bar{V}(t)]^{2}\right\rangle_{t}-\left[\langle\bar{V}(t)\rangle_{t}\right]^{2}$

$\sigma_{V_{i}}^{2}=\left\langle\left[V_{i}(t)\right]^{2}\right\rangle_{t}-\left[\left\langle V_{i}(t)\right\rangle_{t}\right]^{2}$

where $\langle\ldots\rangle_{t}$ denotes time-averaging. In a network of size $N$, we define

$\chi(N)=\sqrt{\frac{\sigma_{\bar{V}}^{2}}{\frac{1}{N} \sum_{i=1}^{N} \sigma_{V_{i}}^{2}}}$

which varies between 0 and 1 . The central-limit theorem implies that, in the limit $N \rightarrow \infty, \chi(N)$ behaves as:

$\chi(N)=\chi_{\infty}+\frac{\delta \chi}{\sqrt{N}}+O\left(\frac{1}{N}\right)$

In particular, $\chi(N)=1$, if the activity of the network is fully synchronized (i.e., $V_{i}(t)=V(t)$ for all $i$ ), and $\chi(N)=O(1 / \sqrt{N})$ if the network activity is asynchronous. Therefore, $\chi_{\infty}$ is equal to 0 in the asynchronous state while a non-zero value of $\chi_{\infty}$ corresponds to synchrony (Ginzburg and Sompolinsky, 1994; Hansel and Sompolinsky, 1992) and the larger $\chi_{\infty}$ the more synchronized the activity is. Note that $\chi_{\infty}$ is sensitive to the correlations in the spike timing of the neurons as well as in the time course of their membrane potential in the subthreshold range. To estimate $\chi_{\infty}$ for given values of the synaptic coupling and of the noise, we compute $\chi(N)$ in simulations for networks of different sizes (in general $N=1600$ and $N=3200$ ) and we extrapolate the results according to Equation (12).

Robustness of synchrony. To probe the robustness of the synchrony of the activity in the network, we study how it resists to noise in the external input. For a given strength of the coupling, the network is in the asynchronous state $\left(\chi_{\infty}=0\right)$ if the noise is sufficiently strong. When the noise level decreases, the asynchronous state may lose stability and synchrony can appear $\left(\chi_{\infty}>0\right)$. The transition from asynchrony to synchrony can
A

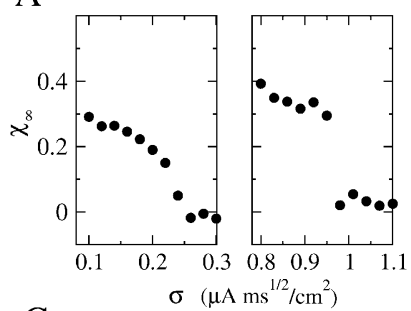

$\mathrm{C}$

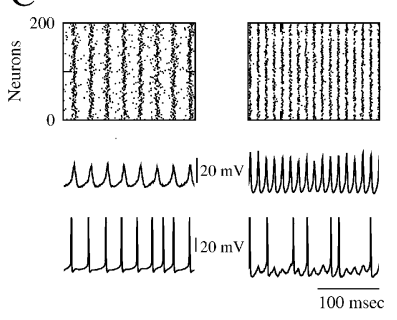

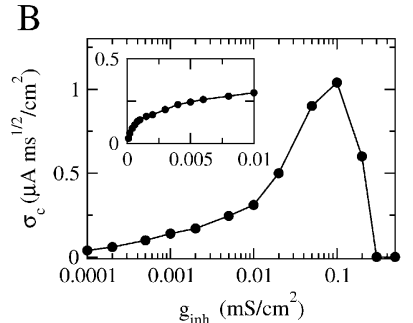

$\mathrm{D}$

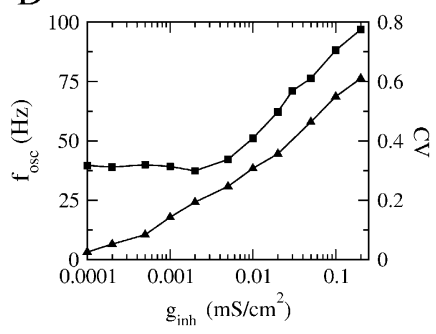

Figure 2. Robustness of collective synchronous oscillations in the network without electrical synapses. (A) The measure of synchrony, $\chi_{\infty}$, vs. the noise. Left panel: $g_{\text {inh }}=0.005 \mathrm{mS} / \mathrm{cm}^{2} ; \sigma_{c}=0.24 \mu A \times \mathrm{ms}^{1 / 2} / \mathrm{cm}^{2}$ Right panel: $g_{\text {inh }}=0.1 \mathrm{mS} / \mathrm{cm}^{2} ; \sigma_{c}=0.98 \mu A \times \mathrm{ms}^{1 / 2} / \mathrm{cm}^{2} ;$ in a small region in the vicinity of $\sigma_{c}$ bistability occurs, that is, the asynchronous state is stable and coexists with a synchronous state (not shown). (B) The criti-

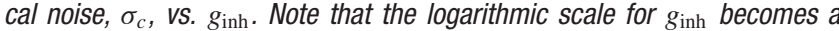
linear one in the inset figure. (C) Patterns of synchrony of the inhibitory network. Left panel: weak inhibition and weak noise $\left(g_{\text {inh }}=0.005 \mathrm{mS} / \mathrm{cm}^{2}\right.$; $\sigma=0.1 \mu \mathrm{A} \times \mathrm{ms}^{1 / 2} / \mathrm{cm}^{2}$ ) Right panel: strong inhibition and strong noise; $g_{\text {inh }}=0.1 \mathrm{mS} / \mathrm{cm}^{2}$ and $\sigma=0.8 \mu A \times \mathrm{ms}^{1 / 2} / \mathrm{cm}^{2}$. Top panels: The raster plots representing the spike trains of 200 neurons. Middle panels: the neuronal membrane potential averaged over all the cells. Bottom panels: The voltage trace of one neuron. (D) The frequency of the population rhythm (squares) and the population average coefficient of variation (CV) of the interspike interval distribution vs. $g_{\text {inh }}$ (triangles). The value of noise is adjusted to be slightly larger than $\sigma_{c}\left(g_{\text {inh }}\right)$. In all the panels, the input current has been adjusted such that the average firing rate of the neurons is kept to $40 \mathrm{~Hz}$ (for $\sigma \approx \sigma_{c}$ ) while changing $g_{\text {inh }}$.

take place in various ways. The most common ways are supercritical or subcritical Hopf bifurcations (Strogatz, 1994). If the bifurcation is supercritical, $\chi_{\infty}$ varies continuously (see Figure $2 A$, left panel for an example) and, at the leading order, it is expected to behave in the vicinity of the bifurcation as:

$$
\begin{aligned}
\chi_{\infty} & =A\left(\sigma_{c}-\sigma\right)^{\alpha} \text { for } \sigma<\sigma_{c} \\
& =0 \text { for } \sigma>\sigma_{c}
\end{aligned}
$$

An example is shown in Figure 2A, left panel. As a matter of fact, fitting the results shown in this figure according to Equations (13) and (14) yield a critical exponent $\alpha=0.4 \pm 0.15$. A similar value for this critical exponent was found at the synchronization-desynchronization transition in the model studied by Brunel and Hansel (2006). These values are compatible with the mean-field prediction, $\alpha=1 / 2$ (Kuramoto, 1984). Hence, we assume $\alpha=1 / 2$ in all the fits performed in this work to estimate $\sigma_{c}$. In contrast, if the bifurcation is subcritical, $\chi_{\infty}$ varies discontinuously from 0 to a non-zero value at some critical value $\sigma_{c}$ (see Figure 2A, right panel). Moreover, the asynchronous state and the synchronous state exist and are both stable in some region near $\sigma_{c}$. In both cases, $\sigma_{c}$ characterizes the robustness of the synchronous state. The smaller $\sigma_{c}$ is, the less robust is the synchrony. 


\section{RESULTS}

The robustness of synchrony solely with inhibitory interactions

The synchronization properties of the network in the case the neurons interact solely via inhibition are depicted in Figure 2. In the left panel of Figure $2 A$, the synchrony measure, $\chi_{\infty}$, is plotted as a function of the noise level, $\sigma$, for an inhibitory conductance $g_{\text {inh }}=0.005 \mathrm{mS} / \mathrm{cm}^{2}$. For this strength of the inhibitory coupling, $\chi_{\infty}$ decreases continuously and is very close to 0 for $\sigma>0.25 \mu \mathrm{A} \times \mathrm{ms}^{1 / 2} / \mathrm{cm}^{2}$. By fitting these data according to Equation (14), one finds that $\sigma_{c} \approx 0.25 \mu \mathrm{A} \times \mathrm{ms}^{1 / 2} / \mathrm{cm}^{2}$. In contrast, for larger coupling $\left(g_{\text {inh }}=0.1 \mathrm{mS} / \mathrm{cm}^{2}\right), \chi_{\infty}$ varies discontinuously from a non-zero value $\left(\chi_{\infty}>0.3\right)$ to nearly zero. The synchronous activity is now more robust since $\sigma_{c} \approx 0.96 \mu \mathrm{A} \times \mathrm{ms}^{1 / 2} / \mathrm{cm}^{2}$. These two examples illustrate the fact that the robustness of the synchrony is significantly affected by the strength of the inhibition.

The dependence of the robustness to synchrony on $g_{\text {inh }}$ is studied more systematically in Figure 2B. When the inhibitory conductance is small, $\sigma_{c}$ varies proportionally with the square root of the inhibitory conductance; see inset. This behavior is consistent with the weak coupling theory of synchronization in large networks of coupled oscillators, which predicts that in the limit of weak coupling the stability of the asynchronous state depends on the ratio $g / \sigma^{2}$ (Pfeuty et al., 2003, see also below). As $g_{\text {inh }}$ increases, $\sigma_{c}$ increases and reaches a maximum at $g_{\text {inh }} \approx 0.1 \mathrm{mS} / \mathrm{cm}^{2}$, for which $\sigma_{c} \approx 1 \mu \mathrm{A} \times \mathrm{ms}^{1 / 2} / \mathrm{cm}^{2}$. Beyond that point, $\sigma_{c}$ decreases and vanishes for $g_{\text {inh }} \approx 0.21 \mathrm{mS} / \mathrm{cm}^{2}$ (corresponding to IPSPS of 1.5 $\mathrm{mV}$ ), indicating that for such strong coupling the network is unable from developing synchronous activity even in the absence of external noise. This non-monotonic behavior, which at first sight is non-intuitive, stems from the sparse connectivity of the inhibition. When the coupling becomes strong, the spatial fluctuations in the connectivity induces strong spatial and temporal fluctuations in the synaptic current, which prevents the network to develop a stable oscillatory activity (Golomb and Hansel, 2000).

The pattern of synchrony that emerges when the noise level $\sigma \lesssim$ $\sigma_{c}\left(g_{\text {inh }}\right)$ depends on $g_{\text {inh }}$. This is shown in Figure $2 \mathrm{C}$ where we compare the raster plot, the population average membrane potential of the neurons, and the trace of one neuron for two values of $g_{\text {inh }}$ (same values as in Figure 2A). For $g_{\text {inh }}=0.005 \mathrm{mS} / \mathrm{cm}^{2}$ and $\sigma=$ $0.1 \mu \mathrm{A} \times \mathrm{ms}^{1 / 2} / \mathrm{cm}^{2}$, the neurons tend to fire one action potential per cycle of the population rhythm. In contrast, for $g_{\text {inh }}=0.1 \mathrm{mS} / \mathrm{cm}^{2}$ and $\sigma=0.8 \mu \mathrm{A} \times \mathrm{ms}^{1 / 2} / \mathrm{cm}^{2}$, a given neuron does not fire at each cycle but with a probability which varies in time and follows the population rhythm.

These two regimes of synchrony also appear clearly in Figure 2D where the frequency of the population rhythm and the coefficient of variation of the interspike histogram distribution computed for each neuron and averaged over the network are plotted as a function of $g_{\text {inh. }}$. For $g_{\text {inh }}<0.005 \mathrm{mS} / \mathrm{cm}^{2}$, the frequency of the population activity oscillations emerging at $\sigma$ slightly larger than $\sigma_{\mathrm{c}}$ and the average firing rate of neurons are both about $40 \mathrm{~Hz}$. Moreover, the spike trains of the neurons are weakly irregular since $\mathrm{CV}<0.2$. In contrast, for $g_{\text {inh }}>0.005 \mathrm{mS} / \mathrm{cm}^{2}$ the frequency of the population rhythm is larger than the population average firing rate. Although the latter does not vary (when $g_{\text {inh }}$ varies, we adjust $I_{\text {ext }}$ to keep the firing rate about $40 \mathrm{~Hz}$ for $\sigma \approx \sigma_{\mathrm{c}}$ ), the frequency of the oscillation increases with $g_{\text {inh }}$. The discharge of the neurons is also more irregular. In the first regime when inhibitory conductances are low, the network displays spike-to-spike synchrony, whereas in the second regime in the presence of strong enough inhibitory conductances and noise, it displays stochastic synchrony (Brunel and Hakim, 1999; Brunel and Hansel, 2006; Tiesinga and Jose, 2000).

\section{Combining electrical and inhibitory synapses}

The simplest and the most intuitive way electrical and inhibitory synapses are likely to combine their effect on the robustness of synchrony is as follows. If both interactions promote synchrony, synchronous activity will be more stable when both interaction types are present than if one of them is blocked. In contrast, if electrical synapses impede synchrony, oscillations will be less stable when both interactions are present than when inhibition acts alone. This type of behavior can be proven in the limit of weak coupling and weak noise under the assumption that the asynchronous state loses stability with a supercritical Hopf bifurcation. Let us denote by $\lambda$ the largest eigenvalue of the the network dynamics linearized around the asynchronous state. At the leading order in the couplings and the noise, $\lambda$ can be expanded (Kuramoto, 1984):

$\lambda=a_{\text {inh }} g_{\text {inh }}+a_{\text {gap }} g_{\text {gap }}-b \sigma^{2}+$ higher order terms

The constant coefficients $a_{\text {inh }}$ and $a_{\text {gap }}$ depend on the single neuron properties and on the connectivities, $K_{\text {inh }}$ and $K_{\text {gap }}$, respectively. The coefficient $b$, which depends on the intrinsic properties of the neurons, is always positive. The asynchronous state is stable when $\lambda<0$. It is unstable if $\lambda>0$ and the robustness, $\sigma_{c}$, is determined by the condition $\lambda\left(\sigma_{c}\right)=0$.

Let us assume first that electrical and inhibitory synapses both promote synchrony. This means that, in the absence of noise, the asynchronous state is unstable if only one of these interactions is present. Therefore, $a_{\text {inh }}$ and $a_{\text {gap }}$ are positive. Hence, for electrical (resp. inhibitory) synapses alone, $\sigma_{c}^{\text {gap }}=\sqrt{a_{\text {gap }} / b}$ (resp. $\sigma_{c}^{\text {inh }}=\sqrt{a_{\text {inh }} / b}$ ). When both synapses are present, the robustness $\sigma_{c}^{\text {gap }+ \text { inh }}$ satisfies the relation:

$\left(\sigma_{c}^{\text {gap }+ \text { inh }}\right)^{2}=\left(\sigma_{c}^{\text {gap }}\right)^{2}+\left(\sigma_{c}^{\text {inh }}\right)^{2}$

We have checked that this equation holds in the numerical simulations of our model when the electrical synapses are sufficiently weak. In particular, Equation (16) implies that:

$\sigma_{c}^{\text {gap }+ \text { inh }}>\sigma_{c}^{\text {inh }}$

In contrast, when the electrical synapses do not synchronize, $a_{\text {gap }}<0$ and $\sigma_{c}^{\text {gap }}=0$. In this case, Equation (15) implies that:

$\sigma_{c}^{\text {gap }+ \text { inh }}<\sigma_{c}^{\text {inh }}$

Therefore, in that case, the robustness is always smaller in the presence of electrical synapses than when they are absent.
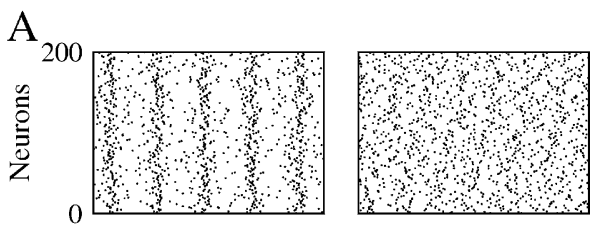

B
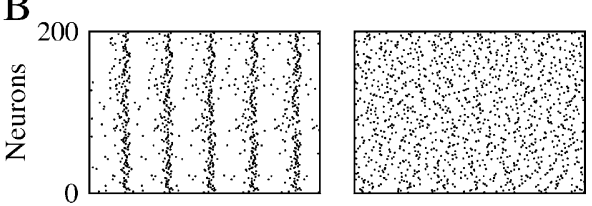

C
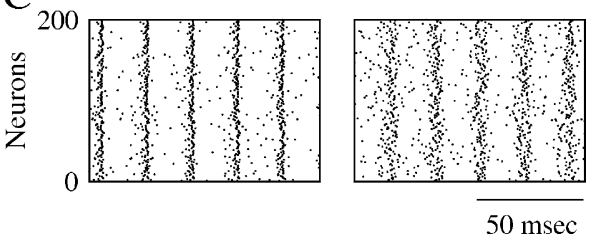

Figure 3. Electrical synapses and inhibitory synapses "sum" their synchronizing effect. Left panels: raster plot representing the activity of 200 neurons from the network in the absence of noise. Right panels: raster plot in the presence of noise; $\sigma=0.5 \mu \mathrm{A} \times \mathrm{ms}^{1 / 2} / \mathrm{cm}^{2}$. (A) $g_{\text {gap }}=0.001 \mathrm{mS} / \mathrm{cm}^{2}$; $g_{\text {inh }}=0$. (B) $g_{\text {inh }}=0.001 \mathrm{mS} / \mathrm{cm}^{2} ; g_{\text {gap }}=0$. (C) $g_{\text {gap }}=0.001 \mathrm{mS} / \mathrm{cm}^{2}$; $g_{\text {inh }}=0.001 \mathrm{mS} / \mathrm{cm}^{2}$. The average number of both types of synapses are $K_{\text {inh }}=K_{\text {gap }}=50$. 

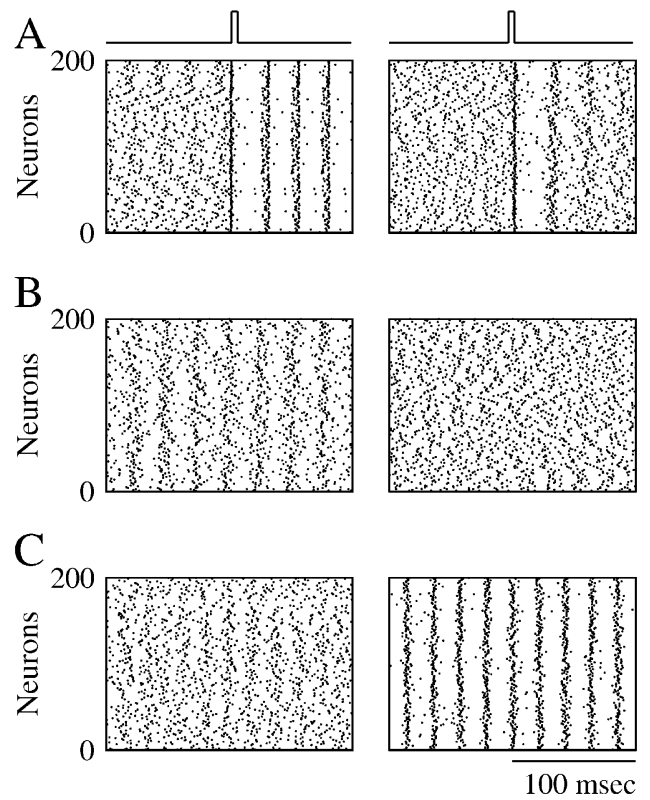

Figure 4. Dendritic electrical synapses improve synchrony only when combined with strong inhibition. Raster plot representing the activity of 200 neurons in the network in which the neurons interact via inhibition and electrical synapses located on their dendrites. (A) $g_{\text {inh }}=0, \sigma=0$. The initial conditions are such that the neurons spike asynchronously. At a given time, a transient pulse of current resets the state of all the neurons and synchronizes their activity. Left panel: when $g_{\text {gap }}=0$ synchrony persists once the resetting pulse is over. Right panel: when $g_{\text {gap }}=0.02 \mathrm{mS} / \mathrm{cm}^{2}$, synchrony dies out in less than $100 \mathrm{~ms}$. (B) $g_{\text {inh }}=0.002 \mathrm{mS} / \mathrm{cm}^{2}, \sigma=0.15 \mu \mathrm{A} \times \mathrm{ms}^{1 / 2} / \mathrm{cm}^{2}$. Left panel: $g_{\text {gap }}=0$; the network activity is synchronized. Right panel: $g_{\text {gap }}=0.02 \mathrm{mS} / \mathrm{cm}^{2}$; synchrony is suppressed. (C) $g_{\text {inh }}=0.01 \mathrm{mS} / \mathrm{cm}^{2}$, $\sigma=0.4 \mu \mathrm{A} \times \mathrm{ms}^{1 / 2} / \mathrm{cm}^{2}$. Left panel: $g_{\text {gap }}=0$; the activity is asynchronous. Right panel: $g_{\text {gap }}=0.02 \mathrm{mS} / \mathrm{cm}^{2}$; the activity is now synchronized.

Equation (16) is not correct beyond the weak coupling limit. However, we expect that Equations (17) and (18) should still hold when the interactions are not too large. An example is shown in Figure 3 when the electrical synapses located on the soma have a conductance $g_{\text {gap }}=0.001 \mathrm{mS} / \mathrm{cm}^{2}$ and an average connectivity, $K_{\text {gap }}=50$, while the maximal inhibitory conductance is $g_{\text {inh }}=0.001 \mathrm{mS} / \mathrm{cm}^{2}$ with average connectivity $K_{\text {inh }}=50$. The left panels of Figures 3A and 3B show that in the absence of noise both type of synapses promote synchrony $\left(\chi_{\infty}^{\text {gap }} \approx 0.25, \chi_{\infty}^{\text {inh }} \approx 0.40\right.$ ) while the left panel in Figure 3C shows that when the interactions are combined the degree of synchrony increases $\left(\chi_{\infty}^{\text {gap }+ \text { inh }} \approx 0.55\right)$. The right panels in Figure 3 correspond to the case where a substantial noise is present in the external input. Here, neither electrical nor inhibitory synapses are sufficient to achieve synchronous activity. However, synchrony occurs when they are combined.

Figure 4 corresponds to a case where the electrical synapses are located on the dendrite and have a conductance $g_{\text {gap }}=0.02 \mathrm{mS} / \mathrm{cm}^{2}$. Such electrical synapses desynchronize the activity of the network. This is shown in the right panel in Figure 4A which was obtained in simulations where the neurons interact only via these dendritic electrical synapses and in the absence of noise. For $t<t_{\text {pulse }}$, the activity in the network is asynchronous. At time $t_{\text {pulse }}$, a strong transient pulse of external current is applied to all the neurons in the network. This pulse resets the states of the neurons which fire synchronously immediately after it, but once the pulse is over synchrony is rapidly lost. This is in contrast with what happens for completely decoupled neurons where synchronous firing does not decay after the transient (Figure 4A, left panel). Hence, electrical synapses destroy synchrony in an "active way." This stems

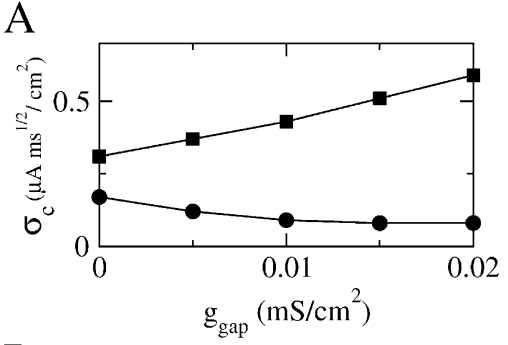

$\mathrm{B}$

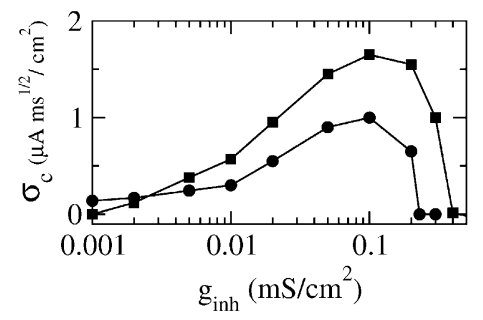

Figure 5. Inhibition dependent potentiation of the effect of electrical synapses on synchrony. (A) $\sigma_{c}$ vs. $g_{\text {gap }}$ for fixed $g_{\text {inh }}$; squares: $g_{\text {inh }}=0.01 \mathrm{mS} / \mathrm{cm}^{2} ;$ circles: $g_{\text {inh }}=0.002 \mathrm{mS} / \mathrm{cm}^{2}$. (B) $\sigma_{c} v s . g_{\text {inh }}$ for fixed $g_{\text {gap }}=0.02 \mathrm{mS} / \mathrm{cm}^{2}$ (squares) and $g_{\text {gap }}=0$ (circles); other parameters as in Figure $2 B$; the external input is adjusted such that the average firing rate of neurons is $40 \mathrm{~Hz}$ (when $\sigma \approx \sigma_{c}$ ).

from the fact that the post-synaptic potentials (PSP) they induce is filtered by the dendrite. When they reach the soma of the postsynaptic cell the PSPs are broadened and delayed (Figure 1B). As a result, these dendritic electrical synapses promote asynchronous firing, that is, they stabilize the asynchronous state (Pfeuty et al., 2005; Saraga et al., 2006). Adding these synapses to weak inhibition reduces the robustness of synchrony as predicted by Equation (18). This is shown in Figure 4B for $g_{\text {inh }}=0.002 \mathrm{mS} / \mathrm{cm}^{2}$. In the absence of electrical synapses, the network settles in a state of partial synchrony (left panel, $\chi_{\infty} \approx 0.2$ ). When electrical synapses $\left(g_{\text {gap }}=0.02 \mathrm{mS} / \mathrm{cm}^{2}\right)$ are also present, synchrony is destroyed.

However, when inhibition is strong, a non-intuitive result is found. The same electrical synapses can actually promote synchrony and improve its robustness to noise. This is shown in Figure $4 \mathrm{C}$ for $g_{\text {inh }}=0.01 \mathrm{mS} / \mathrm{cm}^{2}$. When the neurons interact solely with inhibition, activity is asynchronous because of the large amount of noise (Figure 4C, left panel). However, the synchrony measure increases to $\chi_{\infty} \approx 0.35$, when electrical synapses are also present between the neurons (Figure 4C, right panel).

These results show that the effect of electrical synapses on synchrony can be modulated by inhibition. This modulatory effect also appears clearly in Figure $5 \mathrm{~A}$ where $\sigma_{c}$ is plotted as a function of $g_{\text {gap }}$ for two values of $g_{\text {inh }}$. For the lower value $\left(g_{\text {inh }}=0.002 \mathrm{mS} / \mathrm{cm}^{2}\right), \sigma_{c}\left(g_{\text {gap }}\right)$ is a decreasing function of $g_{\text {gap }}$. In other words, in this case electrical synapses impede synchrony as they do without inhibition. In contrast, for $g_{\text {inh }}=0.01 \mathrm{mS} / \mathrm{cm}^{2}, \sigma_{c}\left(g_{\text {gap }}\right)$ increases with $g_{\text {gap }}$ indicating that strong enough inhibition impacts the effect of the electrical synapses which now promote synchrony. To quantify further how this potentiation of the synchronizing effect of electrical synapses depends on the inhibitory conductance, we plot in Figure 5B the robustness $\sigma_{c}$ as a function of $g_{\text {inh }}$ for $g_{\text {gap }}=0$ and $g_{\text {gap }}=0.02 \mathrm{mS} / \mathrm{cm}^{2}$. The electrical synapses reduce the robustness of synchrony for $g_{\text {inh }}<0.003 \mathrm{mS} / \mathrm{cm}^{2}$, but they increase it for $g_{\text {inh }}$ larger than that value. In particular, although in the absence of electrical synapses synchrony is impossible for $g_{\text {inh }}>0.2 \mathrm{mS} / \mathrm{cm}^{2}$, adding electrical synapses enlarges the synchrony domain up to $g_{\text {inh }}=$ $0.4 \mathrm{mS} / \mathrm{cm}^{2}$. 


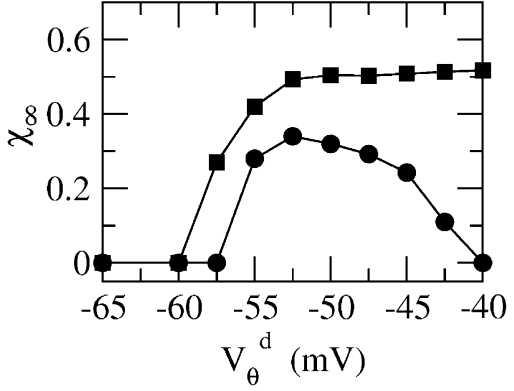

Figure 6. The mechanism underlying the potentiation by inhibition of the effect of electrical synapses on synchrony. The electrical coupling has been modified so that it induces a current only if the membrane potential at the presynaptic site is below a given threshold $\left(V_{\theta}^{\mathrm{d}}\right)$ for dendritic coupling. The synchrony is measured as a function of $V_{\theta}^{\mathrm{d}}$ for small and a large value of the inhibitory conductance. Circles: $g_{\text {inh }}=0.002 \mathrm{mS} / \mathrm{cm}^{2}$, squares: $g_{\text {inh }}=$ $0.01 \mathrm{mS} / \mathrm{cm}^{2}$. For each of these two values, the noise is adjusted so that the network settles close to the transition to synchrony when $g_{\text {gap }}=0$ (see Figure 2A). Also, for each values of $g_{\text {inh }}$ and $V_{\theta}^{\mathrm{d}}$ the external current is adjusted to make neurons fire at $40 \mathrm{~Hz}$. In the presence of weak inhibition, $\chi_{\infty}$ varies non-monotonously with $V_{\theta}^{\mathrm{d}}$ and vanishes for $V_{\theta}^{\mathrm{d}}=-40 \mathrm{mV}$. In contrast, in the presence of strong inhibition, $\chi_{\infty}$ grows monotonously and subsequently saturates.

\section{The mechanism underlying the inhibitory dependent potentiation} of the synchronizing properties of electrical synapses

To analyze further the mechanism behind this cooperative interplay between electrical and inhibitory synapses, let us consider two neurons $A$ and $B$ coupled reciprocally by an electrical synapse. The effect of neuron A on the timing of the firing of neuron $B$ can be qualitatively split into two contributions. One contribution is due to the synaptic current when the membrane potential of neuron $A$ is subthreshold. The other contribution comes from the action potentials fired by $A$ which induces spikelets in $B$. As shown by Pfeuty et al. (2003), although the first contribution helps the two neurons to fire in synchrony, the interaction mediated by the spikelets favors or opposes synchrony depending on the location of the synapse (proximal or distal to the soma) and on the intrinsic properties of the neurons. The overall interaction promotes or impedes synchrony of the two neurons depending on the balance between these two contributions. The threshold and the suprathreshold contributions can be estimated in our model. To this end, we modified the electrical coupling between the two neurons in such a way that there is a current only if the voltage of $A$ is below a value $V_{\theta}$ at the site of the synapse.

Figure 6 depicts for two values of $g_{\text {inh }}$ how, in the presence of dendritic electrical synapses, $\chi_{\infty}$ changes as $V_{\theta}^{\mathrm{d}}$ varies from -65 to $-40 \mathrm{mV}$. In order to compare the effect of electrical synapses for similar levels of synchrony, the noise was set to the value $\sigma_{\mathrm{c}}$ associated with these two values of inhibitory conductances. In the presence of weak inhibition, $\chi_{\infty}$ is very small when $V_{\theta}^{\mathrm{d}} \approx-65 \mathrm{mV}$. This is because the dendritic membrane potential of a neuron is always larger than its resting potentia and, therefore, there is no synaptic current due to the electrical coupling. As $V_{\theta}^{\mathrm{d}}$ increases, $\chi_{\infty}$ increases because the slow subthreshold component of the interaction becomes more effective. However, when $V_{\theta}^{\mathrm{d}} \approx-50 \mathrm{mV}$, $\chi_{\infty}$ reaches a maximum and begins to decrease to zero. This is because the fast suprathreshold component of the interaction is now transmitted via the electrical synapses and it impedes synchrony. In contrast, when inhibition is strong, $\chi_{\infty}$ does not decrease beyond $V_{\theta}^{\mathrm{d}}=-50 \mathrm{mV}$. This means that the desynchronizing effect of the suprathreshold component of the electrical interaction is suppressed.

This analysis clarifies the mechanism by which inhibition modulates how electrical synapses affect synchrony. Strong enough inhibition

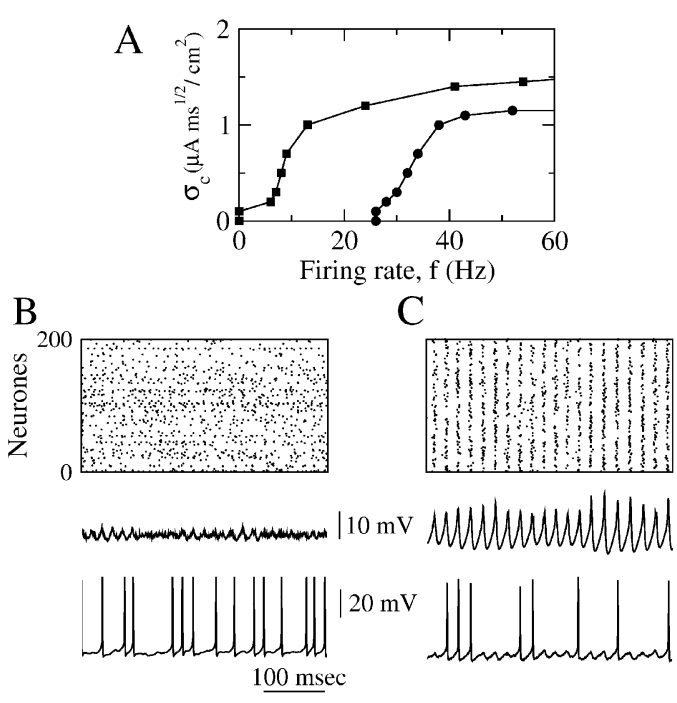

Figure 7. When inhibition is strong, dendritic electrical synapses extend the stability domain of synchrony to low neuronal firing rates. The conductance of inhibitory synapses is fixed at $g_{\text {inh }}=0.1 \mathrm{mS} / \mathrm{cm}^{2}$. (A) $\sigma_{c} v$. the average firing rate of the neurons. Circles: $g_{\text {gap }}=0$. squares: $g_{\text {gap }}=$ $0.02 \mathrm{mS} / \mathrm{cm}^{2}$; the firing rate is varied by changing $I_{\text {ext }}$. (B) Raster plot, population average membrane potential and trace of the membrane potential of one neuron in the network in the absence of dendritic electrical synapses $\left(g_{\text {gap }}=0\right)$. (C) Same as in $\boldsymbol{B}$ but when the electrical synapses are present. In $\boldsymbol{B}$ and $\boldsymbol{C}, I_{\mathrm{ext}}=2 \mu \mathrm{A} / \mathrm{cm}^{2}$ and $\sigma=0.5 \mu \mathrm{A} \times \mathrm{ms}^{1 / 2} / \mathrm{cm}^{2}$.

suppresses the possible desynchronizing effect of the spikelets. As a consequence, in the presence of sufficiently strong inhibition, electrical synapses will always increase the robustness of synchronous firing.

\section{Enhancement of stochastic synchrony at low firing rates by dendritic electrical synapses}

Stochastic synchronous oscillations occur in networks of randomly connected inhibitory neurons in the strong coupling regime. However, their fragility increases when external input and, concomitantly, the average firing rate of the neurons are low. Here, we show that electrical synapses enlarge the range of firing rate where stochastic synchrony is stable even if, when alone, they promote asynchronous firing.

The results depicted in Figure 7 were obtained with $g_{\text {inh }}=$ $0.1 \mathrm{mS} / \mathrm{cm}^{2}$ which corresponds to an IPSP amplitude of $\approx 0.9 \mathrm{mV}$. Figure 7A shows how $\sigma_{c}$ varies as a function of the average firing rate of the neurons (controlled by changing the external current). In the absence of electrical synapses (circles in Figure 7A), synchrony is much less robust at low firing rates than at large firing rates. As a matter of fact, synchrony occurs only if the average firing rate is above $25 \mathrm{~Hz}$. Adding electrical synapses with $g_{\text {gap }}=0.02 \mathrm{mS} / \mathrm{cm}^{2}$ extends the range of firing rates for which synchrony is possible (squares in Figure 7A). This effect of the electrical synapses is also shown in Figures $7 \mathrm{~B}$ and $7 \mathrm{C}$ where the average firing rate of the neurons is about $11 \mathrm{~Hz}$. The raster plot indicates that, with inhibition alone, the activity is asynchronous (left panel). In the presence of electrical synapses $\left(g_{\text {gap }}=0.02 \mathrm{mS} / \mathrm{cm}^{2}\right)$, the network, in contrast, displays synchronous oscillations $\left(\chi_{\infty} \approx 0.54\right)$ at a frequency $\approx 40 \mathrm{~Hz}$ whereas the neurons fire irregularly $(\mathrm{CV} \approx 0.7)$ at an average firing rate that is four times smaller.

The phase diagram of the network is plotted in Figure 8 as a function of $g_{\text {inh }}$ and the average population firing rate when the neurons interact solely via inhibition (Panel A) or via inhibition and dendritic electrical synapses (Panel B). The average population firing rates were varied by changing $I_{\mathrm{ext}}$ with a fixed noise level $\left(\sigma=0.5 \mu \mathrm{A} \times \mathrm{ms}^{1 / 2} / \mathrm{cm}^{2}\right)$. In the white region, 


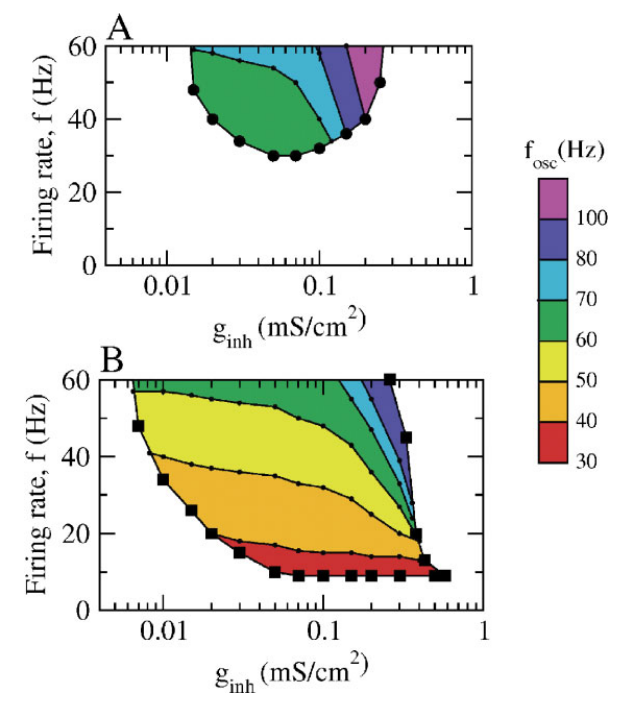

Figure 8. The state of the network as a function of the average firing rate of the neurons and the inhibitory conductance. Population activity oscillations are found in the colored region only. In the white region, the asynchronous state is stable. In the two panels, $\sigma=0.5 \mu \mathrm{A} \times \mathrm{ms}^{1 / 2} / \mathrm{cm}^{2}$. (A) Phase diagram when $g_{\text {gap }}=0$. The network displays synchronous activity with a frequency of population oscillations in the $\gamma$ range only when the average firing rate of the neurons is larger than $35 \mathrm{~Hz}$. (B) Phase diagram in the presence of dendritic electrical synapses. The domain of oscillations extends up to the low activity region.

the asynchronous state is stable. In the colored region, it is unstable and the network settles in a state of synchronous oscillations at a frequency indicated by the color code. Without electrical synapses, the averaging firing is larger than $30 \mathrm{~Hz}$ and the frequencies of the network oscillations larger than $60 \mathrm{~Hz}$. When the dendritic electrical synapses are present, the domain of stability of the collective oscillations extended toward much lower firing and network oscillations frequencies. Note that when the two types of synapses are present, collective oscillations at frequencies around $30-40 \mathrm{~Hz}$ occur in a broad range of inhibitory conductances $\left(0.008-0.5 \mathrm{mS} / \mathrm{cm}^{2}\right)$ even if the individual firing rates are as low as $8 \mathrm{~Hz}$.

\section{DISCUSSION}

\section{Inhibitory synapses potentiate the synchronization properties of} gap junctions

Recent theoretical works have shown that, depending on the intrinsic properties of neurons, the firing frequency of neurons, the location of synapses or the sparseness of the network, electrical synapses can destroy the synchrony of neuronal activity rather than promoting it (Chow and Kopell, 2000; Lewis and Rinzel, 2003; Pfeuty et al., 2003, 2005; Saraga et al., 2006). The present work shows that electrical synapses become less versatile when the neurons also interact via inhibition, as they always promote synchrony. This is because of a modulatory effect of inhibition which "renormalizes" the synchronization properties of electrical synapses. This effect was depicted here in the case of distal electrical synapses which when alone are desynchronizing. We found that this is significant for spiketo-spike synchrony as well as for stochastic synchrony. Similar modulatory effects occur when the electrical synapses are desynchronizing because of the intrinsic properties of the neurons or because their connectivity is too sparse. In all these cases, sufficiently strong inhibition can switch the electrical synapses from being desynchronizing to synchronizing. It should be noted that inhibition also potentiates the synchronizing effect of electrical synapses which, when alone, are already synchronizing. In this case, the function $\sigma_{\mathrm{c}}\left(g_{\text {gap }}\right)$ increases more rapidly with $g_{\text {gap }}$ when the inhibition is strong than when it is weak.

\section{Comparison with previous works}

Our paper focuses on the dynamics of large networks of neurons in the case where inhibition promotes synchrony of the neurons. This contrasts with previous theoretical studies which investigated the dynamics of pairs of neurons coupled by both electrical and inhibitory synapses in the case the inhibitory interaction promotes anti-phase locking (Bem and Rinzel, 2004; Nomura et al., 2003; Pfeuty et al., 2005). Those works showed that electrical synapses promoting synchrony were able to improve the stability of the in-phase locking state and, concomitantly, to induce bistability between in-phase and anti-phase locking. However, the effect these authors studied was not cooperative since the degree of synchronization (e.g., measured as the amplitude of the cross-correlogram peak) with combined synapses was always between those occuring when one or the other type of synapses were alone. Similar results have been obtained in experiments via dual recordings in layer IV cortical interneurons (Gibson et al., 2005), layer I interneurons (Merriam et al., 2005), and snail neurons (Bem et al., 2005).

Kopell and Ermentrout (2004) studied the interplay between electrical and inhibitory synapses. They assumed that the inhibition is sufficiently strong to reset the state of the neurons and to synchronize their action potentials over one cycle. However, in the presence of noise and heterogeneities, strong inhibition also induces suppression of the activity of part of the neurons impeding synchrony. Kopell and Ermentrout showed that electrical synapses can hold the subthreshold voltages of the neuron close together between action potentials. This reduces the effect of the heterogeneities and makes the synchrony induced by inhibition more robust. This effect is different from the effect we have found which is an inhibitory-induced modulation of the synchronization properties of the electrical synapses.

Several modeling studies have emphasized the importance of combining electrical and inhibitory synapses to achieve strong and robust synchronization in simulations of large neuronal networks (Bartos et al., 2002; Traub et al., 2001). However, the precise mechanisms by which electrical synapses contribute to the synchrony in the simulations performed was not analyzed in these papers.

\section{Physiological relevance of the cooperative interplay between electrical and inhibitory synapses}

Experimental studies in neocortical and hippocampal preparations have revealed that both electrical and inhibitory synapses are often required to generate synchrony of firing. This has been shown in large ensemble of neurons in vitro (Blatow et al., 2003; Hormuzdi et al., 2001; LeBeau et al., 2002; Traub et al., 2001) as well as in pairs of neurons (Szabadics et al., 2001; Tamas et al., 2000). Our results showing that electrical synapses combined with inhibition improve the robustness of synchrony in a way which, to a large extent, does not depend on their location, is in line with these experimental results.

It has been proposed that fast synchronous rhythmic episodes in the $\gamma$ range (30-100 Hz) observed in cortex and hippocampus, in vivo or in vitro, are generated within local networks of GABAergic inhibitory interneurons (Bartos et al., 2007; Wang and Buzsáki, 1996; Whittington et al., 1995). Although in $\gamma$-oscillations found in slice preparations, interneurons are likely to fire about one action potential per cycle of the rhythm (Cunningham et al., 2003), recent experiments indicate that in $\gamma$-oscillations observed in vivo during specific behavioral states, GABAergic interneurons fire in a more sparse and irregular way, with a probability of firing per cycle which is between 0.1 and 0.6 (Csicsvari et al., 1999; Tukker et al., 2007). These two modes of synchrony are reminiscent of the two regimes described above, namely spike-to-spike synchrony and stochastic synchrony (Subsection The robustness of synchrony solely with inhibitory interactions). Our results suggest that electrical synapses are especially necessary in this latter 
mode of synchronization which would not be stable otherwise because of the low level of activity of the neurons.

\section{CONFLICT OF INTEREST STATEMENT}

The authors declare that the research was conducted in the absence of any commercial or financial relationships that should be construed as a potential conflict of interest.

\section{ACKNOWLEDGEMENTS}

D.H. was supported in part by a NATO PST-CLG (reference 977683), the $\mathrm{ACl}$ "Neurosciences integratives et computationnelles" (Ministère de la Recherche, France) and project SECyT-ECOS A04B04. D.G. was supported by the Binational US-Israel Science Foundation (grant no. 2003019) and by the Israeli Science Foundation (grant no. 311/04).

\section{REFERENCES}

Amitai, Y., Gibson, J. R., Patrick, A., Ho, B., Connors, B. W., and Golomb, D. (2002) Spatial organization of electrically coupled network of interneurons in neocortex. $J$. Neurosci. 22, 4142-4152.

Bartos, M., Vida, I., Frotscher, M., Meyer, A., Monyer, H., Geiger, J. R., and Jonas, P. (2002). Fast synaptic inhibition promotes synchronized gamma oscillations in hippocampal interneuron networks. Proc. Natl. Acad. Sci. USA 99, 13222-13227.

Bartos, M., Vida, I., and Jonas, P. (2007). Synaptic mechanisms of synchronized gamma oscillations in inhibitory interneuron networks. Nat. Rev. Neurosci. 8, 45-56.

Beierlein, M., Gibson, J. R., and Connors, B. W. (2003). Two dynamically distinct inhibitory networks in layer 4 of the neocortex. J. Neurophysiol. 90, 2987-3000.

Bem, T., and Rinzel, J. (2004). Short duty cycle destabilizes a half-center oscillator, but gap junctions can restabilize the anti-phase pattern. J. Neurophysiol. 91, 693-703.

Bem, T., Le Feuvre, Y., Rinzel, J., and Meyrand, P. (2005). Electrical coupling induces bistability of rhythms in networks of inhibitory spiking neurons. Eur. J. Neurosci. 22, 2661-2668.

Blatow, M., Rozov, A., Katona, I., Hormuzdi, S. G., Meyer, A. H., Whittington, M. A., Caputi, A., and Monyer, H. (2003). A novel network of multipolar bursting interneurons generates theta frequency oscillations in neocortex. Neuron 38, 805-817.

Brunel, N., and Hakim, V. (1999). Fast global oscillations in networks of integrate-and-fire neurons with low firing rates. Neural Comput. 11, 1621-1671.

Brunel, N., and Hansel, D. (2006). How noise affects the synchronization properties of recurrent networks of inhibitory neurons. Neural Comput. 18, 1066-110.

Buhl, D. L., Harris, K. D., Hormuzdi, S. G., Monyer, H., and Buzsáki, G. (2003). Selective impairment of hippocampal gamma oscillations in connexin-36 knock-out mouse in vivo. J. Neurosci. 23, 1013-1018.

Chow, C. C., and Kopell, N. (2000). Dynamics of spiking neurons with electrical coupling. Neural Comput. 12, 1643-1678.

Csicsvari, J., Hirase, H., Czurko, A., Mamiya, A., and Buzsáki, G. (1999). Fast network oscillations in the hippocampal CA1 region of the behaving rat. J. Neurosci. 19 , RC20.

Cunningham, M. O., Davies, C. H., Buhl, E. H., Kopell, N., and Whittington, M. A. (2003). Gamma oscillations induced by kainate receptor activation in the entorhinal cortex in vitro. J. Neurosci. 23, 9761-9769.

Deans, M. R., Gibson, J. R., Sellitto, C., Connors, B. W., and Paul, D. L. (2001). Synchronous activity of inhibitory networks in neocortex requires electrical synapses containing connexin36. Neuron 31, 477-485.

Fricker, D., and Miles, R. (2001). Interneurons, spike timing, and perception. Neuron 32 771-774.

Fukuda, T., and Kosaka, T. (2000). Gap junctions linking the dendritic network of GABAergic interneurons in the hippocampus. J. Neurosci. 20, 1519-1528.

Fukuda, T., Kosaka, T., Singer, W., and Galuske, R. A. (2006). Gap junctions among dendrites of cortical GABAergic neurons establish a dense and widespread intercolumnar network. J. Neurosci. 26, 3434-3443.

Galarreta, M., and Hestrin, S. (1999). A network of fast spiking cells in the neocortex connected by electrical synapses. Nature 402, 72-75.

Gibson, J. R., Beierlein, M., and Connors, B. W. (1999). Two networks of inhibitory neurons electrically coupled. Nature $402,75-79$.

Gibson, J. R., Beierlein, M., and Connors, B. W. (2005). Functional properties of electrical synapses between inhibitory interneurons of neocortical layer 4. J. Neurophysiol. 93, 467-480.

Ginzburg, I., and Sompolinsky, H. (1994). Theory of correlations in stochastic neurona networks. Phys. Rev. E50, 3171-3191.

Golomb, D., and Rinzel, J. (1994). Clustering in globally coupled inhibitory neurons. Physica D 72, 259-282.

Golomb, D., and Hansel, D. (2000). The number of synaptic inputs and the synchrony of large sparse neuronal networks. Neural Comput. 12, 1095-1139.
Hansel, D., and Sompolinsky, H. (1992). Synchrony and computation in a chaotic neural network. Phys. Rev. Lett. 68, 718-721.

Hansel, D., and Sompolinsky, H. (1996). Chaos and synchrony in a model of a hypercolumn in visual cortex. J. Comp. Neurosci. 3, 7-34.

Hormuzdi, S. G., Pais, I., LeBeau, F. E. N., Towers, S. K., Rozov, A., Buhl, E., Whittington, M. A., and Monyer, H. (2001). Impaired electrical signaling disrupts gamma frequency oscillations in connexin 36-Deficient Mice. Neuron 31, 487-495.

Kita, H., Kosaka, T., and Heizmann, C. W. (1990). Parvalbumin-immunoreactive neurons in the rat neostriatum: a light and electron microscopic study. Brain Res. 536, 1-15.

Kopell, N., and Ermentrout, B. (2004). Chemical and electrical synapses perform complementary roles in the synchronization of interneuronal networks. Proc. Natl. Acad. Sci. USA 101, 15482-15487.

Kosaka, T., and Hama, K. (1985). Gap junctions between non-pyramidal cell dendrites in the rat hippocampus (CA1 and CA3 regions): a combined Golgi-electron microscopy study. J. Comp. Neurol. 231, 150-161.

Kuramoto, Y. (1984). Chemical Oscillations, Waves and Turbulence (New York, Springer).

Landisman, C. E., Long, M. A., Beierlein, M., Deans, M. R., Paul, D. L., and Connors, B. W. (2002). Electrical synapses in the thalamic reticular nucleus. J. Neurosci. 22, 1002-1009.

LeBeau, F. E. N., Towers, S., Traub, R. D., Whittington, M. A., and Buhl, E. (2002). Fast oscillations networks induced by potassium transient in the rat hippocampus in vivo. J. Physiol. 542, 167-179.

LeBeau, F. E. N., Traub, R. D., Monyer, H., Whittington, M. A., and Buhl, E. H. (2003). The role of electrical signaling via gap junctions in the generation of fast network oscillations. Brain Res. Bull. 62, 3-13.

Lewis, T., and Rinzel, J. (2003). Dynamics of spiking neurons connected by both inhibitory and electrical coupling. J. Comput. Neurosci. 14, 283-309.

Mancilla, J. G., Lewis, T. J., Pinto, D. J., Rinzel, J., and Connors, B. W. (2007). Synchronization of electrically coupled pairs of inhibitory interneurons in neocortex. $J$. Neurosci. 27, 2058-2073.

Mann-Metzer, P., and Yarom, Y. (1999). Electrotonic coupling interacts with intrinsic properties to generate synchronized activity in cerebellar networks of inhibitory interneurons. J. Neurosci. 19, 3298-3306.

Merriam, E. B., Netoff, T. I., and Banks, M. I. (2005). Bistable network behavior of layer I interneurons in auditory cortex. J. Neurosci. 25: 6175-6186

Nomura, M., Fukai, T., and Aoyagi, T. (2003). Synchrony of fast-spiking interneurons interconnected by GABAergic and electrical synapses. Neural Comput. 15, 21792198.

Pfeuty, B., Mato, G., Golomb, D., and Hansel, D. (2003). Electrical synapses and synchrony: the role of intrinsic currents. J. Neurosci. 23, 6280-6294.

Pfeuty, B., Mato, G., Golomb, D., and Hansel, D. (2005). Combined effect of inhibitory and electrical synapses in synchrony. Neural Comput. 17, 633-670.

Saraga, F., Ng, L., and Skinner, F. K. (2006). Distal gap junctions and active dendrites can tune network dynamics. J. Neurophysiol. 95, 1669-1682.

Sik, A., Penttonen, M., Ylinen, A., and Buzsáki, G. (1995). Hippocampal CA1 interneurons: an in vivo intracellular labeling study. J. Neurosci. 15, 6651-6665.

Sloper, J. J. (1972). Gap junctions between dendrites in the primate neocortex. Brain Res. 44, 641-646.

Strogatz, S. (1994). Nonlinear dynamics and chaos (Reading, MA, Addison-Wesley).

Szabadics, J., Lorincz, A., and Tamas, G. (2001). Beta and gamma frequency synchronization by dendritic gabaergic synapses and gap junctions in a network of cortical interneurons. J. Neurosci. 21, 5824-5831.

Tamas, G., Buhl, E. H., Lorincz, A., and Somogyi, P. (2000). Proximally targeted GABAergic synapses and gap-junctions precisely synchronize cortical interneurons. Nat. Neurosci. 3, 366-371.

Tiesinga, P. H., and Jose, J. V. (2000). Robust gamma oscillations in networks of inhibitory hippocampal interneurons. Network 11, 1-23.

Traub, R. D., Kopell, N., Bibbig, A., Buhl, E. H., LeBeau, F. E. N., and Whittington, M. (2001). Gap junction between interneuron dendrites can enhance synchrony of gamma oscillations in distributed networks. J. Neurosci. 21, 9478-9486.

Traub, R. D., Pais, I., Bibbig, A., LeBeau, F. E., Buhl, E. H., Hormuzdi, S. G., Monyer, H., and Whittington, M. A. (2003). Contrasting roles of axonal (pyramidal cell) and dendritic (interneuron) electrical coupling in the generation of neuronal network oscillations. Proc. Natl. Acad. Sci. USA 100, 1370-1374.

Tukker, J. J., Fuentealba, P., Hartwich, K., Somogyi, P., and Klausberger, T. (2007). Cell type-specific tuning of hippocampal interneuron firing during gamma oscillations in vivo. J. Neurosci. 27, 8184-8289.

Venance, L., Rozov, A., Blatow, M., Burnashev, N., Feldmeyer, D., and Monyer, H. (2000). Connexin expression in electrically coupled postnatal rat brain neurons. Proc. Natl. Acad. Sci. USA 97, 10260-10265.

Wang, X. J., and Buzsáki, J. (1996). Gamma oscillation by synaptic inhibition in a hippocampal interneuronal network model. J. Neurosci. 16, 6402-6413.

Whittington, M. A., Traub, R. D., and Jefferys, J. G. R. (1995). Synchronized oscillations in interneuron networks driven by metabotropic glutamate receptor activation. Nature 373, 612-615.

Whittington, M. A., and Traub, R. D. (2003). Interneuron diversity series: inhibitory interneurons and network oscillations in vitro. Trends Neurosci. 26, 676-682. 